\title{
New Vistas in Plaque Control
}

\section{Lakshmy Menon, Jaiganesh Ramamurthy}

\begin{abstract}
Plaque is the soft, tenacious material found on tooth surfaces which is not readilyremoved by rinsing with water. The most clinically observable plaque on the smoothsurfaces of the teeth along the gingival margin may be termed dentogingival plaque. After tooth cleaning, the supragingival plaque along the gingival border of the teethreaccumulates slowly during the following two days. The thickness of the plaqueincreases dramatically after the third day, to a maximum after seven days.Harnessing technical improvements in mechanical and chemical plaque control methodscan lead to benefits that are significant in ways more than just efficient plaque removal.Fundamental research in biological methods may also add to effectiveness of established methods.It can thus be concluded that pioneering research must be done, the fruits of whichmust be spread around to dental practitioners and academicians for speedy adoption.
\end{abstract}

\section{Definition Of Dental Plaque}

Plaque is the soft, tenacious material found on tooth surfaces which is not readilyremoved by rinsing with water. The most clinically observable plaque on the smoothsurfaces of the teeth along the gingival margin may be termed dentogingival plaque.Dentogingival plaque which occurs on the approximal surfaces, apical to the contactpoints, is termed approximal dental plaque. Plaque may be found below the gingivalmargin in the gingival sulcus or in the periodontal pocket and is termed subgingivalplaque. We normally will find nonattaching bacteria in the most apical position of theperiodontal pocket.Dental plaque is defined clinically as a structured, resilient, yellow-grayish substancethat adheres tenaciously to the intraoral hard surfaces, including removable and fixedprosthesis. The plaque as a potential factor in development of periodontal diseases hasbeen well studied and documented in medical literature.

In children and adults up to 40 years of age, interproximal dental plaque is mainlysubgingival because the gingival papilla normally fills up the interproximal space. In spiteof some loss of periodontal attachment, the papilla will fill up the interproximal spacedue to edema.After tooth cleaning, the supragingival plaque along the gingival border of the teethreaccumulates slowly during the following two days. The thickness of the plaqueincreases dramatically after the third day, to a maximum after seven days.

\section{Prevention Of Periodontal Disease}

There is overwhelming evidence indicating that complete removal of bacterial plaquefrom the dentogingival region is the most effective method of preventing gingivitis andperiodontitis. Control of periodontal disease by eliminating pathogenic organisms of themicroflora is, as yet, impossible, but it is theoretically an attractive approach.Based on epidemiological studies there is a very strong correlation between thelocalization of dentogingival plaque and periodontal disease. 1.1 Epidemiologicalstudies clearly show that there are certain key-risk-teeth (molars and premolars) andkeyrisk- surfaces (the proximal surfaces of the molars and premolars) $\mathrm{Y}=$ FfLrthermore,13- to 16-year-old Swedish children are the "key-risk age-groups" regarding the

progression of dental caries disease in the permanent dentition. In the same age groupwe find around $20 \%$ "key-risk individuals." Clinical studies carried out by Bj6rn andcoworker have showed that $75 \%$ of all interproximal fillings have overhangs in asubgingival position. Also, they found that the loss of periodontal attachment at thesame tooth-surface was correlated to the size of the o verhang as an effect onincreased plaque retention.Hence, successful caries prevention will also result in the prevention of periodontaldisease.The basic principle for preventive dentistry must be that the preventive measures willgive the most significant effect if we concentrate them on "key-risk age groups," "keyriskindividuals," "key-risk teeth" and "key-risk surfaces." That means preventiveprograms based on plaque control have to be concentrated on those tooth surfaceswhere the risk for development or progress of dental disease is most pronounced in agiven population.

\section{Definition Of Plaque Control}

Plaque control normally means preventive measures aimed at removing dental plaqueand preventing it from recurring. This can be accomplished either mechanically orchemically: sometimes the two procedures are combined. 


\section{Home Care}

"Home care" means the sum effect of motivation, knowledge, oral hygiene instruction,oral hygiene aids and motor skill.Today, tooth brushing and other mechanical cleansing procedures are the most reliablemeans of controlling plaque at home. In Scandinavian countries almost $100 \%$ of theschool-children brush their teeth once or twice a day.A Swedish survey showed that no less than $99.5 \%$ of all adults with their own teeth usea toothbrush as an oral hygiene aid. However, only $70 \%$ of men use a toothbrush dailyfrom the age of 30 , whereas $85-90 \%$ of women use a toothbrush daily. Nonetheless,industrious use of the toothbrush is not synonymous with tooth cleaning. In 1971, Hansen \&Gjermou carried out an investigation with the object of evaluating the plaqueremoving effect of various tooth brushing methods on individual tooth surfaces. Thesubjects of the experiment allowed plaque to accumulate freely for three days.

Then a dental hygienist, using an ordinary toothbrush, tested the cleaning effect of theroll-brushing method, the Bass method, Charter's method, and the effect of aninterspace brush. The cleaning effect expressed in terms of Silness\&LSe's plaque-indexis very modest for all methods of brushing despite the fact that brushing was carried outby a dental hygienist.

Similar findings have recently been discovered in study by Bergenholz and coworkers. There is a strong correlation between brushing frequency and the reduction inplaque/gingivitis on the buccal surfaces. The vast majority of self-taught toothbrushersbegins by scrubbing the buccal surfaces, especially at the frontal region, and rarelyproceeds to the lingual surfaces. Interproximal cleaning is simply non-existent in theself-taught.

Most oral hygiene brochures say that tooth cleaning should begin with the use of atoothbrush and toothpaste on the buccal surfaces, followed by the lingual surfaces ofthe upper jaw teeth. Then the buccal surfaces of the lower jaw teeth should be brushed, and finally, the brush should be used on the lingual surfaces of the lower jaw teeth. Onlythen is interdental cleaning considered appropriate. However, there is no odontologicalnecessity for this cleaning order of individual teeth surfaces. The level of ambition is always greatest at the beginning of a tooth cleaning operation.Moreover, more toothpaste is on the toothbrush in the initial phase and the brushbristles are most rigid. In the buccal region, the alveolar bone is very thin and may even

be absent altogether -- on the buccal surfaces of the canines for example, in the lingualand palatine regions, however, the alveolar bone is normally very strong.In light of all these factors, it is evident that the risk of inflicting traumatic lesions duringtooth cleaning is very great if one decides to begin the operation with the buccalsurfaces of the upper jaw teeth. Moreover, far too many people gradually switch from acorrect bass method to a horizontal scrubbing method as the interval since the time ofinstruction increases. This again increases the risk of tooth cleaning trauma.

\section{Plaque-Control}

Plaque control is defined as the removal of microbial plaque and food debris from theoral cavity. The concept of plaque control is broadly based on factors of mechanicalplaque control and chemical plaque control. The mechanical plaque control is mainlyachieved through tooth brushing either using a manual brush or using a motorized toothbrush or with the help of pressurized water pump system involving the use of waterunder pressure pumped through fine blunt needle or nozzle. The chemical control ofplaque includes organic or inorganic chemicals, which inhibit the accumulation, growthand survival of microbiota and debris.

\section{Mechanical Plaque Control:}

The mechanical plaque control is achieved by instituting the different brushing methods, which helps in the disruption of plaque from sub gingival, marginal and supragingivalregion, hence preventing the maturation of plaque which leads to the expression ofvirulent pathogenic bacteria. Usually the dexterity and motivational level of individual tomaintain the mechanical plaque control decreases with the time factor. The level ofmechanical plaque control achieved at individual level decreases on a time gradient.Periodontal disease is highly prevalent and can affectup to $90 \%$ of the world population.

Since the 1960s the essential role of dental plaque as the etiological agent responsible for periodontal disease, the control of biofilm accumulation on teeth has been the key

to periodontal disease prevention.Tooth brushing and the use of dental floss and other devices to remove bacterial plaquefrom the teeth are the most common ways of removing biofilm. Despite its importantrole in the control of periodontal disease, mechanical plaque control is not properly

practiced by most individuals. 


\section{Power Driven Plaque Control}

Dental plaque is structurally and functionally highly organized biofilm. Plaque biofilmhas the ability to begin reorganization within days of being completely disrupted, andleft undisturbed, can become the source of reinfection of gingivitis and periodontitis.Power driven plaque removal has many benefits, for the periodontal patient.

1. Power brushes with timers enable patients to consistently spend more timeremoving plaque. Brushes such as the Sonicare, Flexicare+,Sonicare for kids and Oral-BProfessional care smart series 5000 have timers built into them for patients to brushuntil the timed sequence is over.

2. Studies have shown that invitro plaque biofilm removal is possible beyond thereach of the bristles due to hydrodynamic shear forces of power brushes.

3. During the initial phase of periodontal therapy, power brushes have a superiorability to reduce supragingival plaque and reduce the level of bleeding on probingcompared to manual brushes.

\section{Different Types Of Powered Tooth Brushes For Plaque Control Andhealthy Gums}

Powered toothbrushes were first introduced commercially in the early 1960s, and havebecome established as an alternative to manual methods of toothbrushing.Powered toothbrushes have been designed with different movements of the bristles. Itis therefore important to assess whether they cause damage to the gingival tissuesduring use.To compare powered toothbrushes with different modes of action, in everyday use, bypeople of any age, in relation to

1. removal of plaque

2. health of gingivae

3. adverse effects.

\section{Chemical Agents In Control Of Dental Plaque In Dentistry: An Overview}

Plaque can be removed by two methods-chemical and mechanical. In the chemicalcontrol of plaque, certain anti-bacterial agents are effective.Five categories of agents for approaches have been considered.

1. Broad spectrum antiseptics.

2. Antibiotics aimed at specific bacteriae.

3. Single or combinations of enzymes that could modify plaque structure or activity.

4. Non-enzymatic dispersing or modifying agents.

5. Agents that could affect bacterial attachment.

Antimicrobial plaque inhibitory agent in mouthwashes or toothpastes used to inhibitbacterial plaque formation and thus to prevent or resolve chronic gingivitis. The degreeof effectiveness of commercial mouthwashes is very variable and depends on thecomposition of both the active and various additional agents within the mouthwash.Uses of anti plaque mouthwash

a) To replace mechanical tooth brushing

b) As an adjunct to normal mechanical oral hygiene in situations where this may be

compromised by discomfort or inadequacies.

\section{Solar Powered Tooth Brush Cleans Using Electrons}

The Soladey-3 ionic tooth brush from Japan apparently busts plaque with electrons thatwork with saliva to remove it from your teeth. A solar panel attached to handle absorbselectrons from light and transmits them to your teeth through ionized water and atitanium oxide semi conductorin the upper shaft of the tooth brush. It is different frommost electric toothbrushes, which vibrate and sometimes shoot water at your teeth, butstill rely on tooth paste to help remove plaque.

\section{How It Works?}

There is a light activated titanium rod (semi conductor) inside the handle. Whenexposed to any light source ( a fluorescent bathroom bulb, a plain light bulb or sunlight), the photo-sensitive titanium rod inside Soladey converts light into negatively chargedions(electrons). The rod releases these ions, which blend with saliva to attract positive(hydrogen) ions from the acid in the dental plaque on your teeth. A systemic review ofthe effectiveness of self-performed mechanical plaque removal in subjects withperiodontal disease concluded that it had limitations. Therefore, adjunctive use ofchemical plaque control might be beneficial.Hence a chemical plaque has to be addressed on individual level on daily basis forproper maintenance of oral health.

\section{Chemical Plaque Control}

Agents are classified into three generations

\section{First Generation}

a)Phenols (Triclosan)7: It's a phenol derivative which is synthesized used as a topical 
antimicrobial agent with a broad spectrum of action including against both gram -ve andgram +ve bacteria. It also has specific action against mycobacterium and candidaspecies.Mechanism of Action: Triclosan acts on cytoplasmic membrane and induce leakage ofcellular contents which leads to bacteriolysis and Cell death.Triclosan is induced in toothpaste to prevent plaque formation. It is used along with zinccitrate or its polymer gantrez to enhance its retention in the oral cavity. It also inhibitsprostaglandins and leukotriens thereby it reduces the degree of inflammation.

Metallic ions: These are $\mathrm{Zn}$-ions and $\mathrm{Cu}$-ions. It acts by reducing the glycolytic activity inbacteria and hence delays bacterial growth.

Quaternary Ammonium Compounds: These are Benzathonium Chloride, BenzalleniumChloride and Cetylpyredinum.

These are cationic antiseptics and surface active agents which are effective against gram+ve organisms.

Mechanism of action: Positively charged molecule reacts with negatively charged cellmembrane phosphates and thereby disrupting the bacterial cell wall structure.

The side effects includes staining and enhanced calculus formation, it also causesburning sensation and desquamation. Sanguinare: Sanguinare chloride is currently usedin both mouth rinses and toothpaste. It is an extract from blood root plant Sanguinarecandensis. It is anbenzophenanthredinealleloid.Mechanism of action is not known. It is most effective against gram positive organisms.

\section{Second Generation}

Bisbiguanides 8 is biguanidesposses anti plaque activityincludingChlorhexidine, Alexidine and Octenidine. Chlorhexidinegluconate, a cationicbisbiguanide is the best known and widely used member of this class. The antiplaqueproperties of chlorhexidine are unsurpassed by other agents.

It has much greater and more prolonged effects than other antiseptics.10 Thedigluconate of chlorhexidine (1: 6 - Di 4 -chlorphenyl - diguanidohexane) is a syntheticantimicrobial drug which is effective in vitro against both gram positive and gramnegative bacteria including aerobes, anaerobes, yeastand fungi.

Mechanism of action: Prevents pellicle formation by blocking acidic groups of salivaryglycoprotein's thereby reducing glycoprotein adsorption on to tooth surface. Preventsadsorption of bacterial cell wall on tooth surface.Prevents binding of mature plaque.Antibacterial action of Chlorhexidineconsits of two actions, i.e., bacteriostatic at lowconcentration and bactericidal at high concentration.

Bacteriostatic action at low concentration is mainly due to the negative energy of thebacterial cell wall reacts with positive energy chlorhexidine molecule. This alters theintegrity of cell membrane and Chlorhexidine binds to inner membrane phospholipidsand increases permeability. This leads to the vital elements leaks out resulting inbacterial cell death.Bactericidal Action is due to the higher concentration of chlorhexidine. This causeprogressive greater damage of membrane and the larger molecular weight compoundsloss and coagulation and precipitation of cytoplasm. The Free CHX molecules enter thecell and coagulate proteins and vital cell activity ceases and cause resultant cell death.It has shown that $0.2 \% \mathrm{CHX}$ mouth rinses will prevent development of experimental gingivitis, it has been shown that Chlorhexidine is more effective in preventing plaqueaccumulation on a clean tooth surface than in reducing pre existing plaque deposits. Theadverse effects of chlorhexidine include brownstaining of tooth and restoration, loss oftaste sensation and stenosis of parotid duct. It affects the mucous membrane andtongue; and may be related to the precipitation of chromogenic dietary factors on teeth

and mucus membrane. It is probable that one cationic group attaches chlorhexidine tothe tooth and mucosal surface, which the other cationic group lyse the bacterial cellwall.Thus the cationic group can also attach dietary factors such as gallic acid derivativesfound in some foods and beverages including tea, coffee and wines.Essential oils11 (Listerine): It's a combination of phenol related essential oil, thymolandmethyl salicylate. It has shownto have moderate plaque inhibitory effect andantigingivitis effect. It has poor oral retention and has burning and bittertaste.

Mechanism of Action: The action of phenol acid is cell wall disruption and inhibition ofbacterial enzyme. The phenolic compound are also known to act as scavengers ofoxygen free radical and hence has an effect on leucocyte activity.

\section{Third Generation}

Delmopinol14 : It's a relatively new preparation which inhibits plaque growth andingivitis. It interferes with plaque matrix formation and also reduces bacterial adhesionand adherence.

It ceases binding of plaque to tooth, thus aiding the easy removal of plaque bymechanical procedures. It is indicated as a pre brushing mouth rinse.Adverse effects ofDelmopinol are staining of tooth and tongue, taste disturbances and mucosal sorenessand erosion. Miscellaneous agents includes Salt of $\mathrm{Zn}$ and $\mathrm{Cu}$, bispyridinecetridine, amylases , proteases and cloxtranase. 
In conclusion all these agents were effective in plaque disruption and preventing itsmaturation. Clinicians should advice their patients after considering the positive effectof each of these agents against a favorable result could derived by theirpatients.As a result, there is a very positive correlation between brushing frequency, gingivalretraction and trauma caused by an abrasive lesion on the buccal surfaces.Up to the age of 25 , the greatest loss of periodontal attachment is found on the buccalsurfaces in the average patient. This is brought about principally by Tooth brushing, andis particularly unfortunate in cases where the bifurcations have been exposed on thebuccal surfaces of the molars, indirectly causing root separation or extraction.A fundamental principle for all preventive action is that the positive effect is greatest

where the risk of disease development is greatest.The patient has the greatest chance of being able to see positive results in his oralhygiene efforts if he concentrates initially on "key-risk teeth" and "key-risk surfaces."After this we can make more stringent oral hygiene demands by including buccalsurfaces where results are largely indiscernible.

A Swedish survey shows that approximately $46 \%$ of adults use toothpicks sporadically.Of these, $12 \%$ use toothpicks daily. On the other hand, dental floss is used irregularly by $12 \%$ of adults, and $2 \%$ of these use dental floss daily. In other words, toothpicks areused six times more frequently than dental floss as an oral hygiene aid by adults. Despite this, it must be recognized that interdental cleaning is practically non-existent asan established habit in most countries. In the light of normal plaque distribution anddental disease in the dentition and on individual tooth surfaces, we can therefore statethat needs-related tooth cleaning does not take place. The aspirations of the adultpatient today focus principally on those tooth surfaces with the least disease formation,and cleaning, therefore, only produces approximately a $20 \%$ effect. In other words,there is a largely unexploited source of dental care here which we must tap. Yet out of

8760 hours per year, the individual patient normally spends no more than two hours inthe dental clinic.

\section{Conclusion}

A perusal through the scientific literature on methods of plaque control makes itMclear that various methods have been tried from time to time. Further, it clarifies that innovations in technology can make older methods less suitable since side effects and causes of inefficiency can gradually be corrected. However, slow pace of spread of novel ideas outside research or academic circles and similar lack of speed in general administration of such methods is seen and it can be surmised that procedures are being done at lower efficiency levels. Harnessing technical improvements in mechanical and chemical plaque control methods can lead to benefits that are significant in ways more than just efficient plaque removal. Fundamental research in biological methods may also add to effectiveness of established methods. It can thus be concluded that pioneering research must be done, the fruits of which must be spread around to dental practitioners and academicians for speedy adoption.

\section{References}

[1] Biological plaque control: novel therapeutic approach to periodontal disease, Naoyuki Sugano, Journal of Oral Science, Vol. 54, No. 1, 1-5, 2012

[2] Emerging concepts in oral chemical plaque control - an overview, George Philip et al, INTERNATIONAL JOURNAL OF DENTA L CLINICS,2012, Volume 4 Issue2

[3] Different powered toothbrushes for plaque control and gingival health (Review), Deacon SA

[4] Powered toothbrushes are more effective than manual toothbrushes in reducing gingival bleeding or inflammation, SUMMARY REVIEW/PERIODONTOLOGY

[5] Tooth brush a key to mechanical Plaque Control, Deepak Grover et al, Review Article Concept and practice of plaque-control, Axelsson, Pediatric Dentistry, 1981 